Editorial

\title{
Quantum Reports: A New Journal for a Broad Audience
}

\author{
Lev Vaidman ${ }^{(0)}$
}

Raymond and Beverly Sackler School of Physics and Astronomy, Physics Department, Tel Aviv University, Tel Aviv 69978, Israel; vaidman@post.tau.ac.il

Received: 27 June 2018; Published: 4 July 2018

A new MDPI journal Quantum Reports is launched as a scientific journal to provide an advanced forum for the growing community of researchers of quantum science. When I finished my studies on the foundations of quantum mechanics about thirty years ago, I had almost no options of finding a university to continue research in this field. Today, it is hard to find a university which has no quantum center and the majority of these centers are very young.

Quantum theory had several revolutions in its history. The birth of quantum physics was the revolution against classical physics, which was on the verge of celebrating a full understanding of nature, but failed to explain newly discovered quantum effects. One may consider the second revolution to be the Einstein-Podolsky-Rosen paper, followed by Bell's proof, which showed that bizarre quantum phenomena cannot be explained by an underlying local classical theory. The next revolution was when technology allowed us to see these bizarre effects in the laboratory. And the current revolution is these quantum effects moving from bizarre paradoxical peculiarities to the core of new technology. Quantum optics experiments move from optical tables to quantum optical chips. Quantum communication reaches outer space. Quantum computers are seriously considered as a threat to classical cryptography, while quantum cryptography is almost ready to replace classical protocols. The new field of quantum materials moves technology to new standards.

In spite of full agreement with experimental results and wide applications, the meaning and interpretation of quantum theory remains open. Questions about the relevance of quantum effects in various phenomena in chemistry and even in biology are under hot discussion. These are legitimate questions, although I would put them somewhat differently. For me, there is no question that everything is quantum. The question is: can the approximation of classical physics be used for describing a particular phenomenon? And if there is a satisfactory classical explanation of some phenomenon, it is not enough. Since the true theory is quantum theory, everything needs to be explained in quantum terms. Apart from the bizarre consequences of quantum theory for reality, the serious problem which questions the validity of quantum theory is the very limited progress in understanding gravity in terms of quantum mechanics. Maybe a new revolution is needed here. And although I personally would prefer the full success of quantum theory, the existence of a fundamental unsolved question makes the field even more attractive to many scientists.

There are many journals with sections for quantum physics and many journals fully devoted to quantum physics. Quantum Reports will compete with them and the publishing experience of MDPI will surely provide many advantages. However, we do not exactly present an alternative to these journals. Many of them are highly specialized and focus on technical aspects of various topics of quantum physics. Quantum Reports aims for a broad audience. Preference will be given to papers with new conceptual advances. It might be a theoretical paper introducing a new paradigm or an experimental paper breaking current technological limits or both a paper reporting an experiment demonstrating new theoretical concepts. The scope is intentionally very broad: we will consider a philosophical paper solving interpretational problems of quantum theory, but also a report on a newly produced 
quantum material. What I hope will make Quantum Reports an important journal is that it aspires to reach high standards, constantly looking for path-breaking results. All articles will be peer-reviewed, but the focus will be on trying to predict its future impact rather than determining whether the work is completely free from technical errors. The first priority is that the work is interesting. The goal of Quantum Reports is to widely and rapidly disseminate new discoveries.

Our broad and expanding Editorial Board is eager to fulfil this task. If you feel that you can contribute, please contact the Editor. The launch of a new journal is an exciting moment and we encourage you to submit your articles. We will make all efforts to ensure that your new, important ideas will bring the next quantum revolution even closer.

(C) 2018 by the author. Licensee MDPI, Basel, Switzerland. This article is an open access article distributed under the terms and conditions of the Creative Commons Attribution (CC BY) license (http://creativecommons.org/licenses/by/4.0/). 